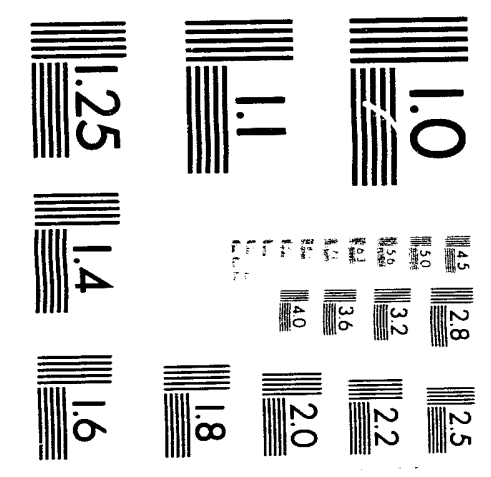



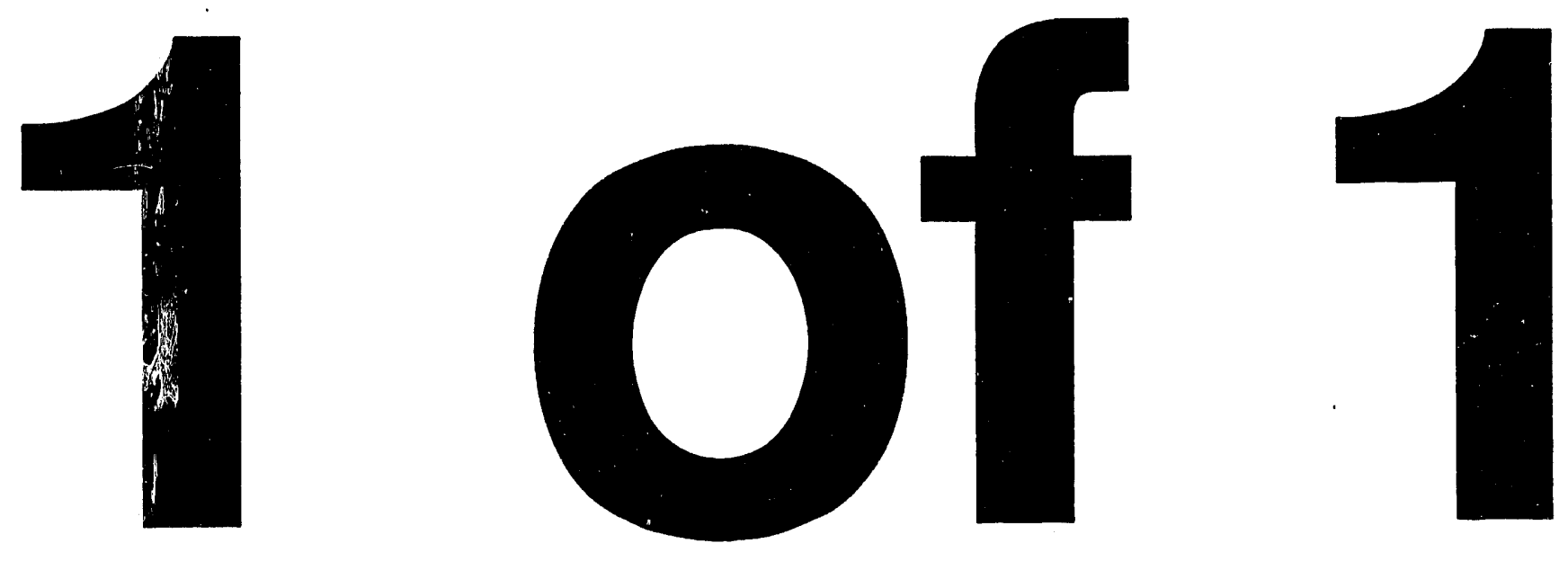


\title{
EFFICIENCY OF ARC DISCHARGE AND BEAM EXTRACTION OF THE DIII-D NEUTRAL BEAM ION SOURCE
}

\author{
by \\ R. HONG
}

This is a preprint of a paper to be presented at the 15th IEEE Symposium on Fusion Engineering, October 11-15, 1993, Cape Cod, Massachusetts, and to be printed in the Proceedings.

\author{
Work supported by \\ U.S. Department of Energy \\ Contract DE-AC03-89ER51114
}
GENERAL ATOMICS PROJECT 3466
OCTOBER 1993
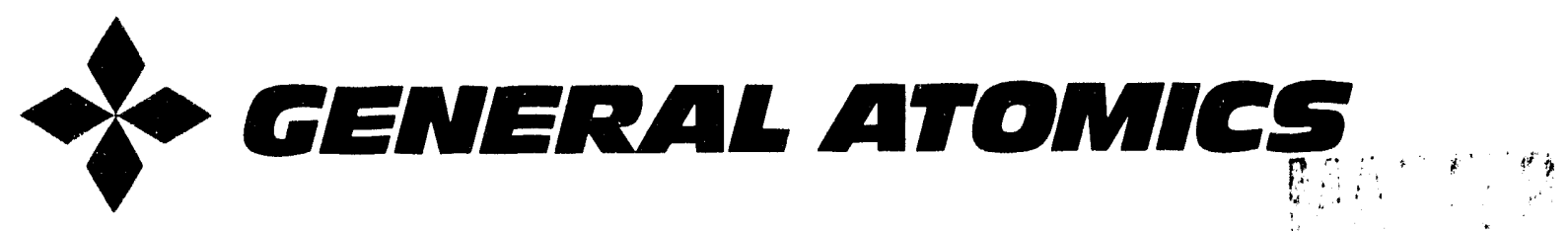


\title{
EFFICIENCY OF ARC DISCHARGE AND BEAM EXTRACTION OF THE DIII-D NEUTRAL BEAM ION SOURCE
}

\author{
R. Hong \\ General Atomics \\ P.O. Box 85608, San Diego, California 92186-9784
}

\begin{abstract}
DIII-D neutral beam ion sources are operated in the emission limited arc discharge regime. Filament temperature has been observed to play an important role in the ion source operation [1]. Effects of the filament temperature on the arc discharge efficiency (Langmuir probe signal of the arc discharge/arc power) and arc efficiency (extracted beam current/arc power) have been experimentally measured. The results show that both eficiencies reach optimum values with respect to filament temperature. The optimum arc efficiency is independent of the beam energy, but the filament temperature at which optimum arc efficiency is obtained shifts to higher values with higher beam energy. Gas puff into the neutralizer is necessary to achieve optimum neutralization efficiency, but it also increases both particle collisions in the accelerator column and gas flow into the arc chamber. Tests show that arc efficiency decreases only slightly with increasing particle collisions, since loss in the extracted beam current is compensated for by the decreasing arc power required to sustain the arc discharge with additional gas flow. The most significant effect of
\end{abstract}

particle collisions is the sharp increase of the gradient grid current, which can cause damage to the grid.

\section{INTRODUCTION}

DIII-D neutral beam ion sources (Fig. 1) have been operated for plasma heating with high reliability since 1987. Filament temperature has been observed to play an important role in the operation of these ion sources, which are designed to operate in the emission limited arc discharge regime. Control of the filament temperature becomes crucial in obtaining a stable arc discharge in the arc chamber and beam extraction through the accelerator. This regulation is achieved by regulating the voltage applied to the filaments. In addition to the filament temperature control, an arc regulation technique was developed and has been very successful in regulating the arc discharge and the extracted beam current. This arc regulation capability provides opportunities to study ion source performance with various operational parameters. Experiments have been recently performed

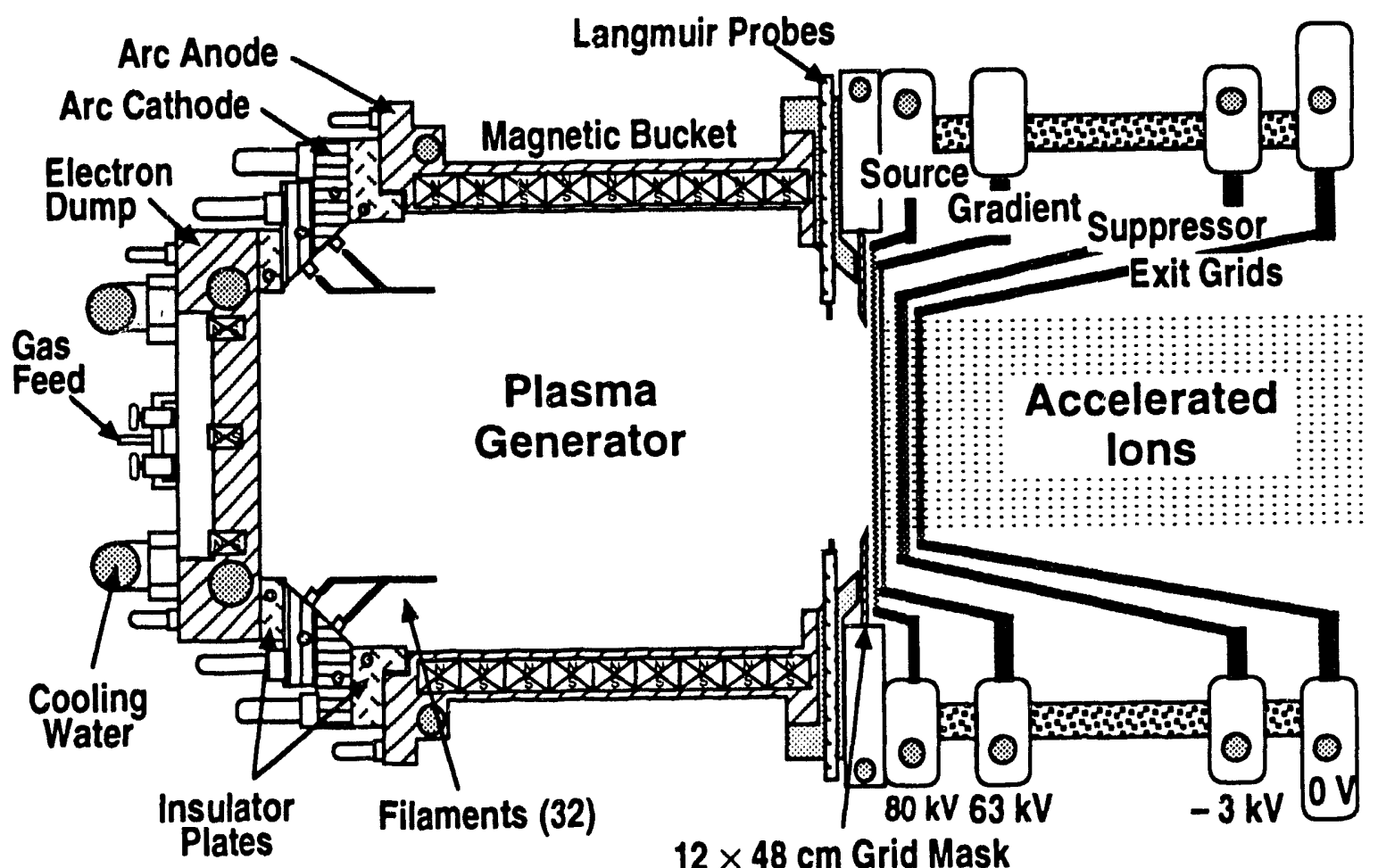

FIG 1. DIII-D neutral beam ion source.

Mamuscript received October 12, 1993. This is a report of work sponsored by the U.S. Department of Energy under Contract No. DE-AC03-89ER51114. 
on one of the ion sources to study the effects of the filament temperature and particle collisions on the efficiency of the arc discharge, the efficiency of beam extraction, and on the beam optics. 1- studying the filament temperature effects, ion source operating parameters such as beam energy, source gas puff rate, Langmuir probe signal, and beam pulse length were all kept constant while varying the voltages applied to the filaments. To study collisional effects on the arc efficiency and beam optics, additional gas was introduced into the neutralizer during the beam pulse while holding other operational parameters constant. These gases diffuse into the accelerator and arc chamber of the ion source. All tests were performed with the ion source operated in deuterium.

\section{ARC EFFICIENCY IS INDEPENDENT OF THE BEAM PULSE LENGTH}

An intrinsic characteristic of the DIII-D neutral beam ion sources is the slow increase of the arc and beam currents during the beam pulse. This increase is attributed to the heating of the filaments by the energetic electrons backstreaming from the accelerator into the arc chamber, resulting in a change of the beam perveance during a beam pulse and possibly an overdense condition. An arc regulation technique [2] was developed to regulate beam current by using Langmuir probe signals for feedback regulation of the ion saturation current of the plasma in the arc chamber. The arc efficiency for various beam pulses was measured and it does not vary with beam pulse length, indicating that the arc regulation circuit is very effective in regulating the extracted beam current. This observation excludes any effect of beam pulse length on the beam extraction, thus making it possible to study the effects of operational parameters on ion source performance. Deuterium beams of $1 \mathrm{sec}$ pulse length were used in the experiments for the rest of this study.

\section{EFFECT OF THE FILAMENT TEMPERATURE ON THE ION SOURCE OPTICS}

An ion source is said to operate at optimum beam perveance (beam perveance is defined by the ratio of the extracted beam current to the beam energy) when minimum gradient grid current (normalized to the extracted beam current) is obtained. The procedure to obtain this optimum beam perveance is called perveance tuning, which measures the normalized gradient grid currents during beam extraction as a function of the arc discharge level (varying the Langmuir probe signal in our system) for a fixed beam energy and filament temperature. The optimum beam perveance for DIII-D ion sources has been measured to be $3.0 \mu \mathrm{p}\left[1 \mu \mathrm{p}=10^{-6} \mathrm{~A} /(\mathrm{V})^{3 / 2}\right]$ with fixed filament temperature, and it is independent of the beam energy. Studies were also performed to determine if optimum beam perveance varies with filament temperatures. Because we have no capability to measure the filament temperature, we use the voltages applied to the filaments as a measure of the filament temperature. Fig. 2 shows that the optimum perveance is independent of the voltage applied to the filaments. This result also implies that filament temperature has no effect on the beam optics, and confirms that the optimum beam perveance is determined by the geometry of the accelerator grids and the voltage gradients between the accelerator grids.

\section{EFFECTS OF THE FILAMENT TEMPERATURE ON THE EFFICIENCY OF THE ARC DISCHARGE AND BEAM EXTRACTION}

It has been observed (Fig. 3) that more ions are extracted from the ion source when the voltage applied to the filaments is increased, although the Langmuir probe signals, which measure the ion saturation current of the arc discharge inside the arc chamber, are regulated at a constant value, and the beam energy is also a constant. A test [3] was performed to measure the electron temperature of the arc discharge inside the arc chamber. Results show that the electron temperature decreases with the applied filament voltage (Fig. 4) resulting in higher particle density since the ion saturation current is kept at a constant value, thus more ions are available for extraction with hotter filaments. The arc efficiency of the ion source optimizes with filament voltage (Fig. 5). The filament voltage at which maximum arc efficiency is obtained depends on the beam energy. Fig. 6 shows that the arc efficiency decreases with the beam perveance when lower voltages are applied, but maintains its optimum value at higher filament voltage. This result is closely related to an observation which shows that the arc discharge efficiency (Langmuir probe signal/arc power) of an ion source that operates in the emission limited discharge regime is sensitive to the filament temperature and is also dependent on the arc power level. It proves

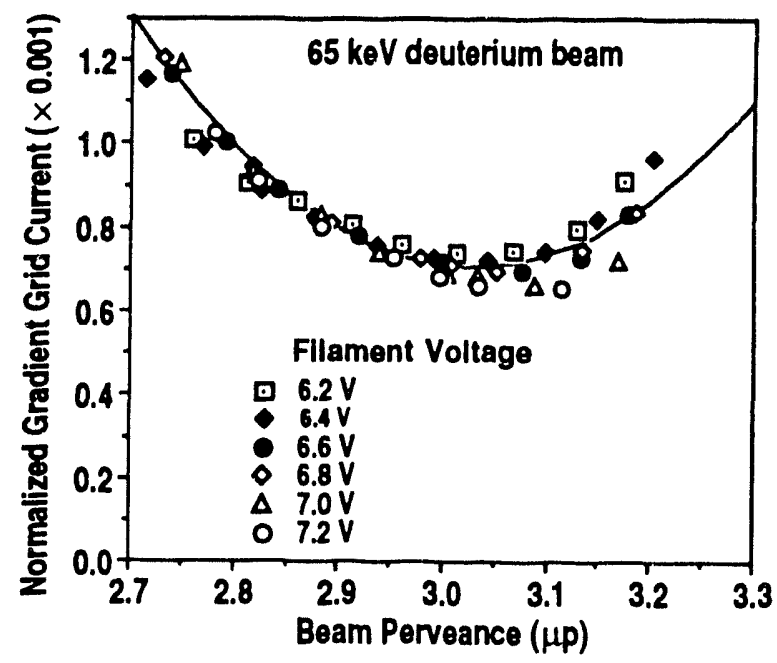

Fig. 2. Filament temperature has no effect on the optimum beam perveance. 


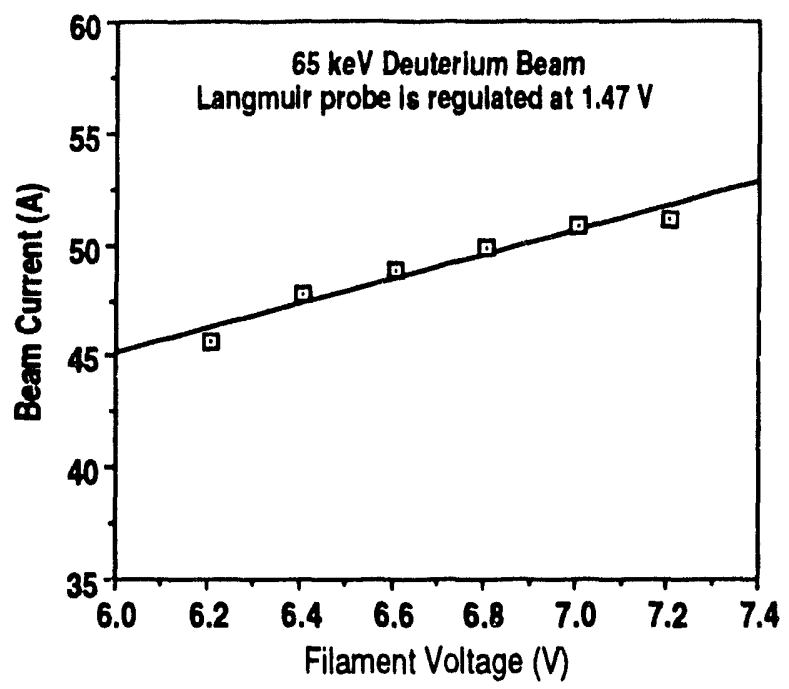

Fig. 3. More ions are available for extraction with hotter filaments.

that filament temperature plays a dominant role in both efficiencies of arc discharge and beam extraction.

\section{EFFECTS OF PARTICLE COLLISIONS ON THE ARC EFFICIENCY AND BEAM OPTICS}

It has been observed [4] that ion source operation is less sensitive to the amount of gas puffed into the ion source than to the filament temperature. The gas flow rate is determined by the following considerations. First, there should be enough gas flow to prevent the gas starvation condition, which will result in decreasing ion beam extracted during the beam pulse. Based on operational experience, a gas puff rate of 20 torr $-l / \mathrm{sec}$ into the arc chamber of the ion source satisfies this requirement. Second, there is a need to puff additional gas into the

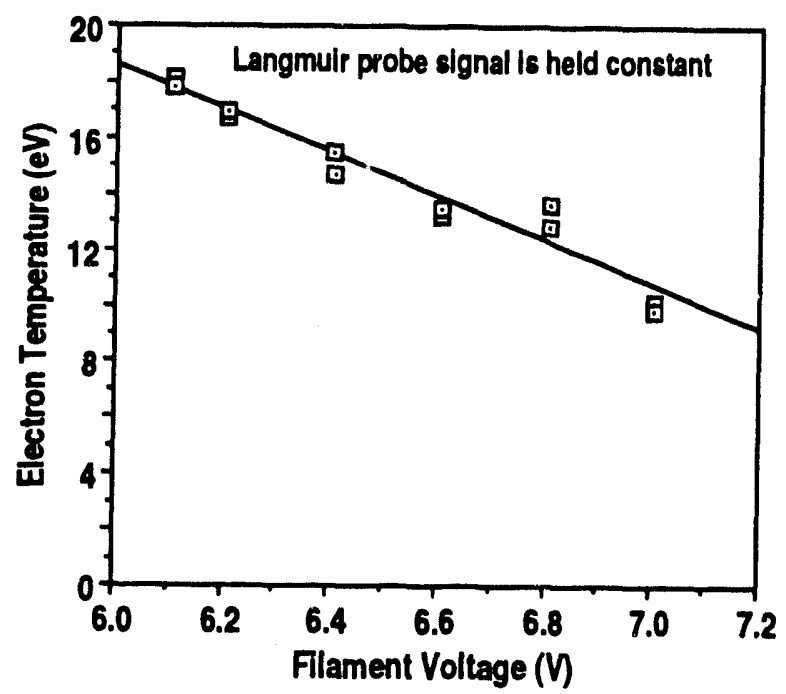

FIG. 4. Electron temperature of the arc discharge decreases with hotter filaments.

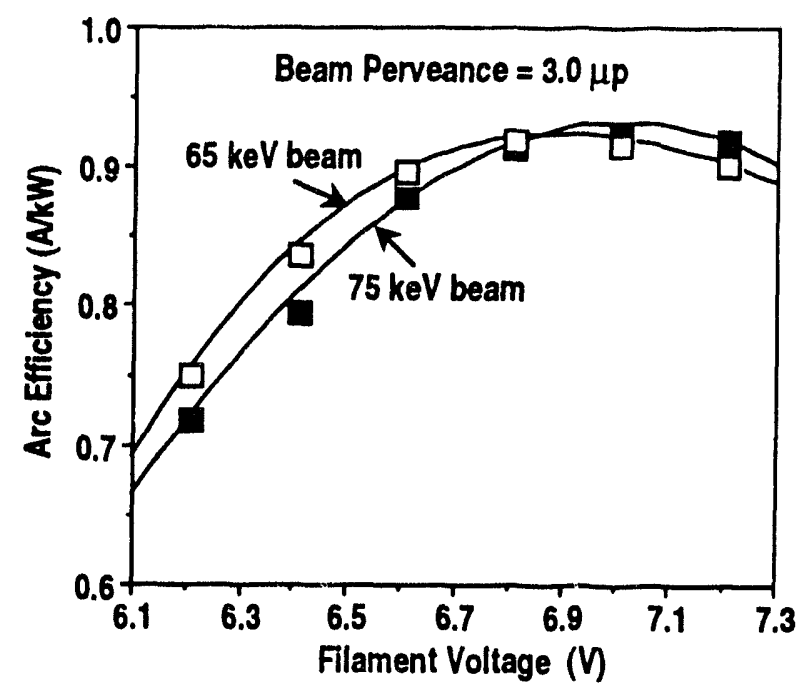

Fig. 5. Arc efficiency as function of the voltage applied to the filaments for two beam energies.

neutralizer during beam extraction to obtain optimum neutralization efficiency for optimum neutral power and to prevent overheating of beamline internal components by the excessive residual energetic ions. Measurements have shown that optimum neutralization efficiency is obtained when 10 torr- $\ell / \mathrm{sec}$ of gas, in addition to the 20 torr- $\ell / \mathrm{sec}$ source gas flow, is puffed into the neutralizer during the beam pulse [5]. Excessive gas fiow into the neutralizer will not further increase the neutralization efficiency, but will significantly increase the particle collisions within the accelerator column when neutral gases diffuse from the neutralizer into the accelerator of the ion source. These particle collisions will affect the beam optics and increase the number of particles deposited on the accelerator grids, which can result in the termination of the beam pulse to protect the accelerator grids from overheating It has been found, Fig. 7, that the gradient grid current, which is the ion source operational signal most sensitive to the beam optics, increases linearly with the neutralizer gas puff. We also found that both the arc power required to sustain a constant arc discharge density inside the arc chamber and the extracted beam current decreased slightly with increased neutralizer gas flow. The small decrease in the required arc power is attributed to the increase of gas diffused from the neutralizer into the arc chamber, making it possible to sustain the same level of arc discharge with less arc power. The decrease in the beam current is due to the collisional loss of energetic ions, but it is a very small amount because the mean free path (about $100 \mathrm{~cm}$ ) of the accelerated ions is very large compared to the size of the accelerator in direction of the beam path (about $2.7 \mathrm{~cm}$ from the first grid to the exit grid of the accelerator). The result is that the arc efficiency shown decreases only very slightly with increasing particle collisions. 


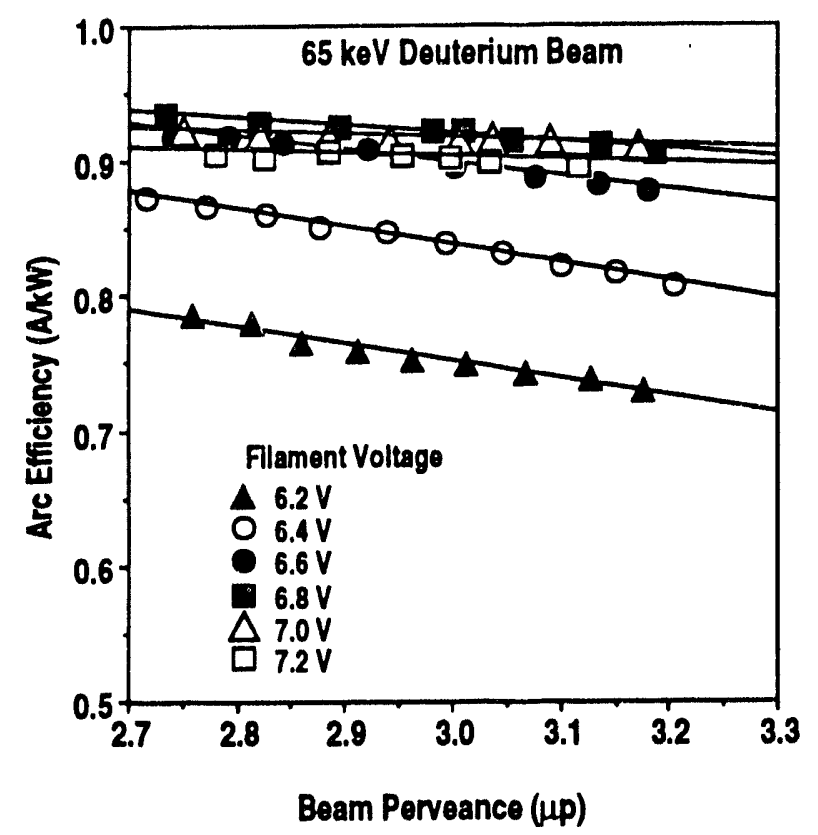

FIG. 6. Arc efficiency versus beam perveance for various filament temperatures.

\section{CONCLUSIONS}

The filament temperature plays an important role in ion source operation. The filament temperature has no effect on the beam optics or on the optimum beam perveance, which is determined by the geometry of the accelerator grids and the voltage gradients between the accelerator grids. With effective arc regulation, the arc efficiency does not vary during the beam pulse, making it possible to study effects of ion source operational parameters on the arc efficiency. Both optimum arc discharge efficiency and beam extraction are obtained at a certain filament temperature which varies slightly with the arc power level and the beam energy. Sufficient gas puffing into the ion source is required to prevent gas starvation which is key to achieving both stable arc discharge and stable beam extraction. Additional gas needs to be introduced into the neutralizer during the beam pulse both to obtain optimum neutralization efficiency and to minimize the number of residual energetic ions to avoid overheating the beamline internal components. Particle collisions do not have a significant effect on the arc efficiency, which decreases very slightly with increasing gas puff into the neutralizer. Based on this study, an operational regime is

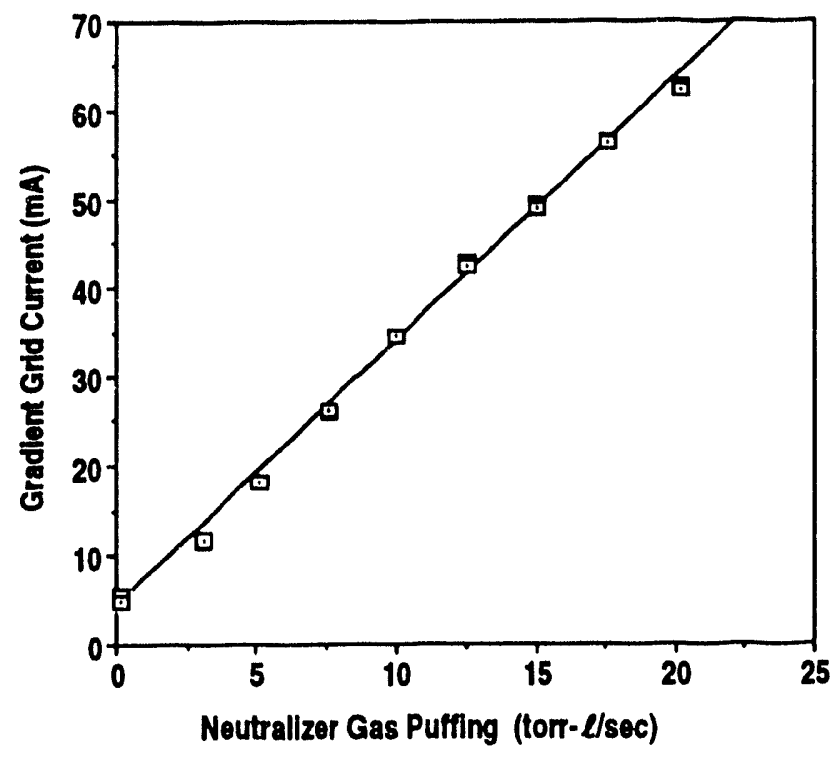

Fig. 7. Gradient grid current increases linearly with the neutralizer gas puff.

obtained for achieving optimum arc efficiency and beam neutralization without the risk of causing damage to the ion source.

\section{ACKNOWLEDGMENT}

The author would like to thank Bonnie Scoville for her assistance in operating the ion source and taking data for this study.

\section{REFERENCES}

[1] R. Hong, A.P. Colleraine, J.S. Haloovec, D.H. Kellman, J. Kim, A. Nerem, et al., "Operational experience with the DIII-D neutral beam $12 \mathrm{~cm} \times 48 \mathrm{~cm}$ common long pulse source," in Proc. 12th Symp. on Fusion Engineering, pp. 1133-1136, 1987.

[2] R. Hong, D. Kellman, G. Santamaria, A. Colleraine, J. Kim, R. Lee, et al., "Beam current regulation of DIII-D neutral beam long pulse ion sources," in Proc. 13th Symp. on Flusion Engineering, pp. 987-890, 1989.

[3] D.H. Kellman, J. Busath, and R. Hong, "Messurements of plasme temperature and density in the DIII-D neutral beam jon source arc chamber," this symposium.

[4] R. Hong, A.P. Colleraine, J. Kim, R. Lee, J. Phillipo, and J. Wight, "DIII-D neutral beam long pulse source operational charecteristics of various source gas flow rates," Bull. Amer. Phys. Soc., vol. 33, p. 2054, 1988.

[5] J. Wight, R.-M. Hong, and J. Phillips, "Recent DIII-D netr tral beam calibration results," in Proc. 14th Symp. on Fusion Engineering, pp. 66-68, 1891. 

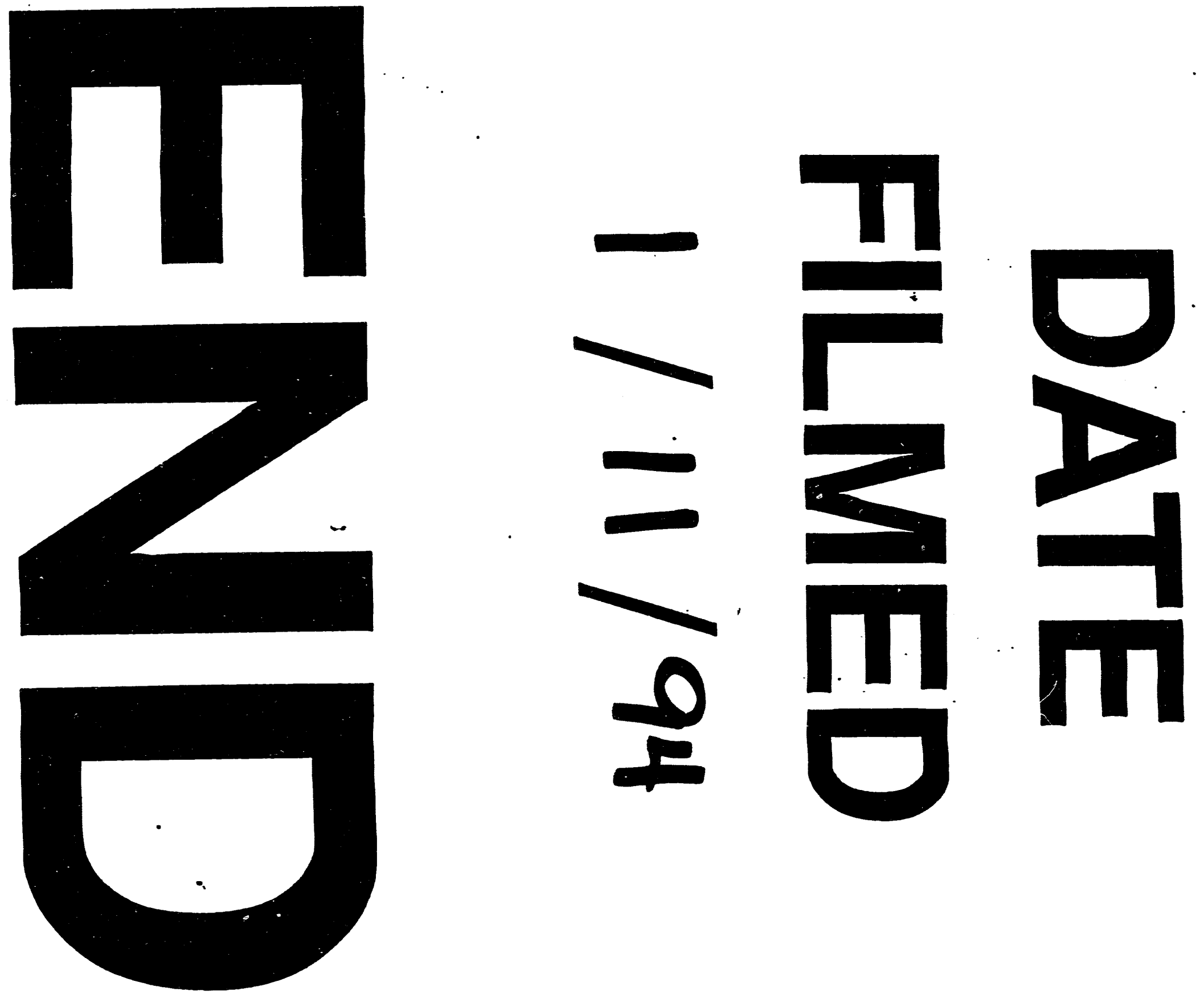

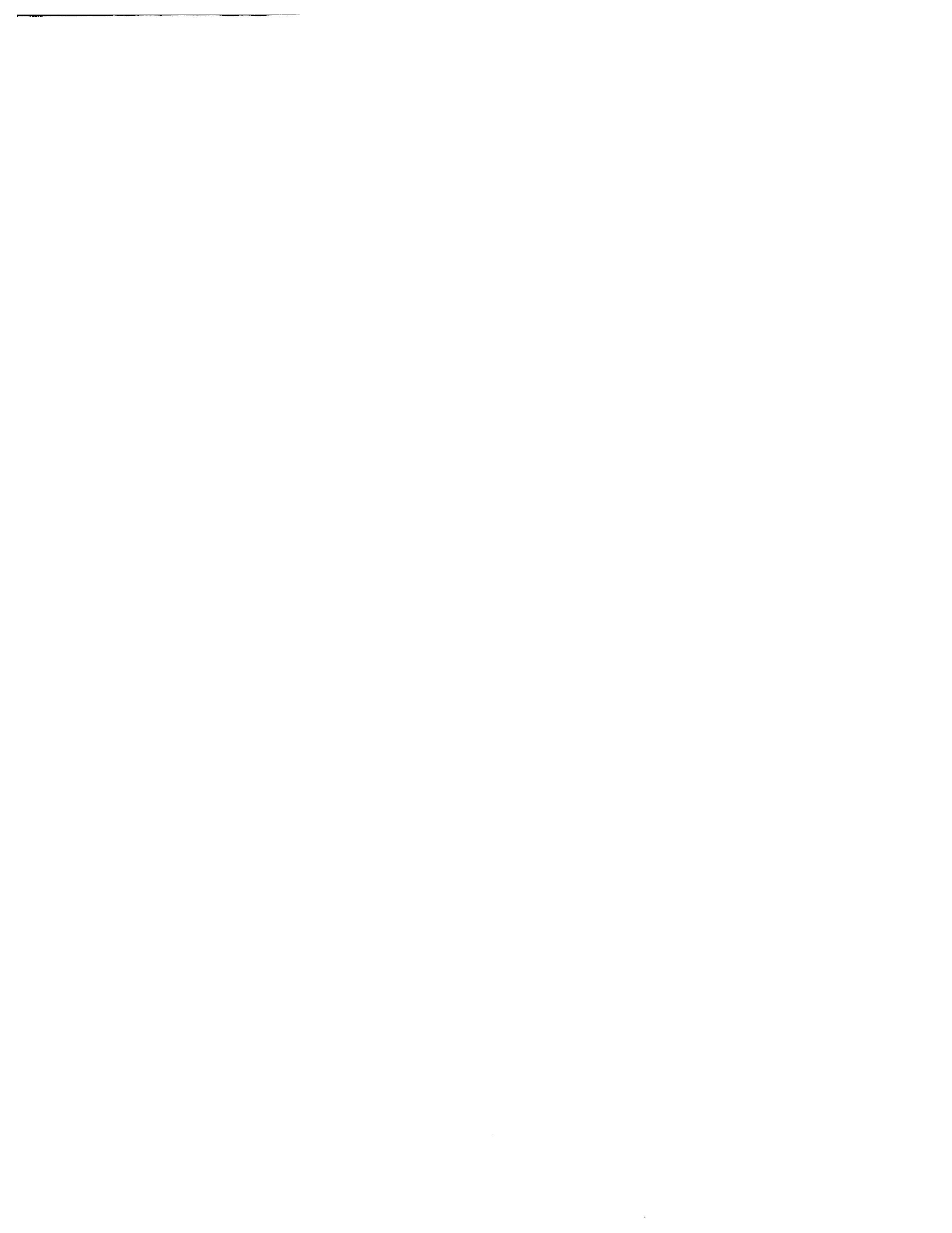